\title{
Streamlining Disease Surveillance System implementation in Tanzania: Lessons Learnt
}

\author{
Green M. Sadru \\ Immunization, John Snow Inc. (JSI), Dar es Salaam, Dar es Salaam, Tanzania, United Republic of \\ Objective
}

To support streamlining of VPD surveillance into integrated diseases surveillance and response (IDSR) system in Tanzania

\section{Introduction}

Tanzania adopted IDSR as the platform for all disease surveillance activities. Today, Tanzania's IDSR guidelines include surveillance and response protocols for 34 diseases and conditions of public health importance, outlining in detail necessary recording and reporting procedures and activities to be taken at all levels. A total of 15 disease-specific programs/sections in the Ministry of Health, Community Development, Gender, Elderly and Children (MOHCDGEC) are linked to the IDSR, though the extent to which each program uses IDSR data varies. Over the years, IDSR procedures and the structures that support them have received significant government and external resources to maintain and strengthen detection, notification, reporting and analysis of surveillance information.

However, with the imminent phasing out of programs (such as the Polio eradication program) that have supported IDSR strengthening and maintenance in the past, resources for surveillance will become more limited and the government will need to identify additional resources to sustain the country's essential surveillance functions.

Maternal and Child Survival Program (MCSP), a USAID Funded Program supported MOHCDGEC managing active and passive surveillance systems in improving coordination and strengthen the system taking into consideration declining resources as well as transitioning to polio end game where most of the financial resources were derived from to support vaccine preventable diseases surveillance. The support complements other Global health security agenda (GHSA) on the key thematic areas (Prevent, Detect and Report) support to the MOHCDGEC and working with the newly formed Emergency Operations Center (EOC) to improve response.

\section{Methods}

Between February and November 2018, the MOHCDGEC and MCSP undertook activities to generate information for future plans to strengthen Tanzania's disease surveillance system to address the Global Health Security Agenda (GHSA): 1) desk review of country's disease surveillance 2) meetings with stakeholders involved in surveillance; 3 ) workshop where stakeholders discussed and developed strategies for streamlining disease surveillance; 4) asset mapping to identify assets (human, financial, physical 5) stakeholders meeting to further discuss and agree on future strategies, activities.

\section{Results}

The Disease surveillance system review found the functions for surveillance being implemented at different levels (Figure 1). These include identifying cases; reporting suspected cases, conditions, or events; investigating and confirming suspected cases, outbreaks and events. To facilitate decision making at different levels, it was found that analysing and response are done at all levels. A total of 15 disease-specific programs/sections in the MOHCDGEC are linked to the IDSR, though the extent to which each program uses IDSR data varies.

\section{Key strengths and opportunities}

The government's adoption of the IDSR platform and the fact that the MOHCDGEC has a dedicated department to monitor IDSR performance has been a great achievement of the program. The system is fully adaptable to support all disease surveillance with clear supervisory structures in place at regional and council levels. At the operational level there is presence of full-time, competent and dedicated government employees and exhibiting awareness of their responsibilities, and resourcefulness. The entire surveillance program benefits from government and external funding for disease-specific surveillance-related programs (e.g. funds for polio eradication and malaria program).

SDS Annual Conference Proceedings 2019. This is an Open Access article distributed under the terms of the Creative Commons AttributionNoncommercial 4.0 Unported License (http://creativecommons.org/licenses/by-nc/3.0/), permitting all non-commercial use, distribution, and reproduction in any medium, provided the original work is properly cited. 
Despite the achievements, there are notable challenges faced by the program including disease-specific programs often requiring additional information and opting to set up parallel surveillance systems rather than integrating with the IDSR; surveillance activities often not being considered high priority at council level relative to curative service and/or surveillance not being a line item in budgets; electronic data transmission platforms not being able to support transmission of all e-IDSR data with the result that health facility data (including diseases for immediate notification) may not get reported in weekly transmissions; high turnover of surveillance staff and unsystematic orientation of newly-deployed staff; discrepancies in reported HMIS, IDSR, and diseasespecific program data indicating data quality issues.

\section{Asset mapping:}

At the time of the review, the number of staff available varied widely between programs, with the national laboratory and the National AIDS Control program (NACP) reporting the highest number at council level and Immunization and Vaccine Development (IVD) having significant number of persons supporting vaccine preventable disease surveillance. At the time of the review, most of the funds were allocated in capacity building through training and supportive supervision compared to core surveillance function.

\section{Key inteventions to streamlining and harmonizing of surveillance}

Supported the roll out of electronic IDSR to ensure real time surveillance through DHIS2

Supported proceedures to establishement of surveillance expert working group (EWG);

Development of Term of reference for EWG to guide implementation of IDSR activities Development of transition plan highlighting key stakeholders and the support they provide to strengthening surveillance in the country;

Development of workplan to guide implementation of agreed recommendations which includes;

1. Coordinating activities of all stakeholders involved in surveillance,

2. Developing or advocating for an interoperable and harmonized reporting system through DHIS2 that will accommodate the needs of the various disease- and event-surveillance programs,

3. Promoting synergies at national level so that active surveillance is expanded as appropriate to other diseases and supports case based surveillance,

4. Building capacity of RHMTs/CHMTs in leadership and management to manage human and financial resources and prioritize surveillance;

5. Coordinating and strengthening disease and event-surveillance at community level by having at least one trained focal person at the community for all disease surveillance.trained focal person at the community for all disease surveillance.

\section{Conclusions}

Streamlining and strengthening of the surveillance system could be achieved by existing coordination structures within MOHCDGEC. Strengthening IDSR by implementing an interoperable of reporting systems including integration of laboratory data will achieve harmonization, consistency in data and appropriate response. At the Regional and council level, priority activities identified include strengthening coordination, orientation and training for financial and human resources management for surveillance aimed at strengthening surveillance and response teams. The IDSR should strengthen active surveillance to adopt case based surveillance as deemed appropriate for more diseases. A proposed plan for implementing key activities to achieve integration and streamlining of disease surveillance has been developed and it is hoped that resources will be made available for immediate implementation.

\section{Acknowledgement}

MCSP Disease surveillance, is a GHSA -funded activity which is co-funded with USAID Maternal and Child Health funding.

SDS Annual Conference Proceedings 2019. This is an Open Access article distributed under the terms of the Creative Commons AttributionNoncommercial 4.0 Unported License (http://creativecommons.org/licenses/by-nc/3.0/), permitting all non-commercial use, distribution, and reproduction in any medium, provided the original work is properly cited. 
Surveillance Functions and data Flow across levels
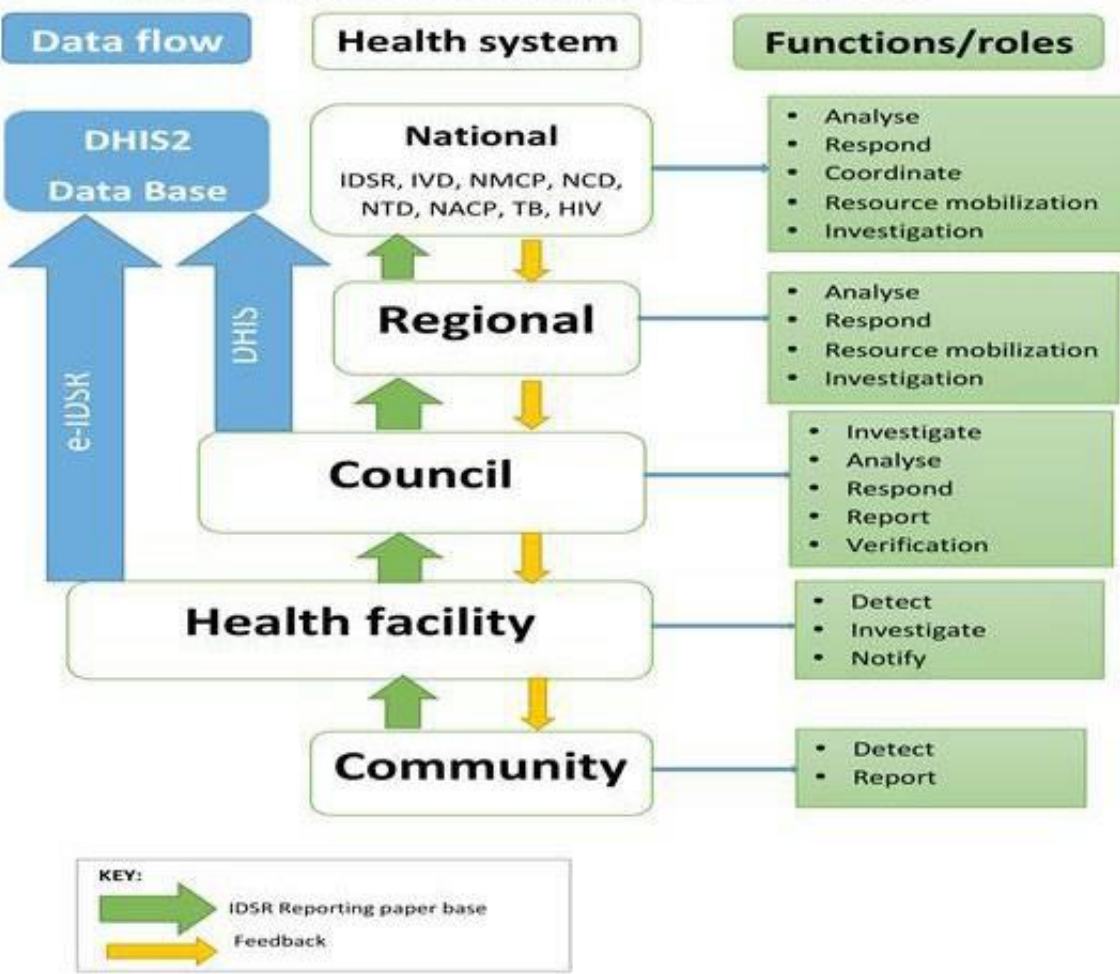